\title{
Intrinsic noise-induced phase transitions: Beyond the noise interpretation
}

\author{
O. Carrillo, ${ }^{1}$ M. Ibañes, ${ }^{1}$ J. García-Ojalvo, ${ }^{2,3}$ J. Casademunt, ${ }^{1}$ and J. M. Sancho \\ ${ }^{1}$ Departament d'Estructura i Constituents de la Matèria, Universitat de Barcelona, Diagonal 647, E-08028 Barcelona, Spain \\ ${ }^{2}$ Departament de Física i Enginyeria Nuclear, Universitat Politècnica de Catalunya, Colom 11, E-08222 Terrassa, Spain \\ ${ }^{3}$ Center for Applied Mathematics, Cornell University, 657 Rhodes Hall, Ithaca, New York 14853
}

(Received 2 December 2002; published 21 April 2003)

\begin{abstract}
We discuss intrinsic noise effects in stochastic multiplicative-noise partial differential equations, which are qualitatively independent of the noise interpretation (Itô vs Stratonovich), in particular in the context of noise-induced ordering phase transitions. We study a model which, contrary to all cases known so far, exhibits such ordering transitions when the noise is interpreted not only according to Stratonovich, but also to Itô. The main feature of this model is the absence of a linear instability at the transition point. The dynamical properties of the resulting noise-induced growth processes are studied and compared in the two interpretations and with a reference Ginzburg-Landau-type model. A detailed discussion of a different numerical algorithm valid for both interpretations is also presented.
\end{abstract}

DOI: 10.1103/PhysRevE.67.046110

PACS number(s): 64.60.Cn, 05.40.-a, 02.50.Ey

\section{INTRODUCTION}

An important feature of nonlinear systems is their ability to sustain organized behavior even in the presence of a substantial amount of randomly fluctuating influences. Even more strikingly, systems which in the absence of fluctuations exhibit a disordered behavior can experience, under certain conditions, the emergence of spatiotemporal order upon addition of a suitable amount of noise [1]. The most basic manifestation of this fact is the existence of ordering phase transitions induced by noise in dynamical systems with spatial degrees of freedom $[2,3]$. These transitions bring the system from a disordered to an ordered phase as the intensity of the noise increases, contrary to naive intuition. By disordered (ordered) phase we mean for example the homogeneous zero (nonzero) state corresponding to the coarse graining of a spin field with random (uniform) orientation.

Ordering phase transitions are usually driven by multiplicative noise terms, which depend on the system's variables [4]. But the stochastic integrals associated with stochastic differential equations with multiplicative noise are not uniquely defined [5]. Among the many interpretations that can be given to these integrals, two are frequently used: the Stratonovich interpretation that follows the standard rules of calculus, but gives rise to nonintuitive statistical properties of the noise terms, and the Itô interpretation that avoids these problems, but at the expense of requiring new rules of calculus.

Beyond the technical mathematical definitions, the physical implications of both noise prescriptions boil down to an important fact. The Stratonovich prescription for white noise yields the result one would get for a time-correlated noise in the limit of vanishing correlation time. The key point is that, as soon as the noise is slightly correlated, the stochastic variables defined by the corresponding Langevin equation build up correlations with the noise variable at equal time. This immediately implies that the multiplicative noise terms in the equation have a nonzero mean, even with a zero-mean noise. The result is the so-called Stratonovich drift, a net force induced by noise which is at the heart of most noise-induced phenomena, in particular, concerning noise-induced ordering transitions.

As for a given stochastic differential equation with multiplicative noise, the results do depend on the interpretation, a preliminary analysis of the physical problem has to be performed to make a judicious choice. Our experience indicates that there are a minimum of three possible situations.

(1) If we start with a well established deterministic differential equation and some controlled parameter is allowed to fluctuate (experimental or realistic external noise), one would always expect the noise to have a high-frequency cutoff and as a consequence the Stratonovich interpretation is usually argued to be the reasonable choice.

(2) If the starting scheme is a master equation which is approximated by a Fokker-Planck equation, then one can write a stochastic differential equation with multiplicative noise in the Itô interpretation. This happens, for instance, in front propagation problems on a lattice [6].

(3) Moreover, quite often our initial scheme is a set of stochastic differential equations, and we would like to simplify the problem eliminating the most irrelevant fast variables (those with a very short-time scale). The interpretation of the final stochastic differential equation will depend on the order, in which this procedure is performed with respect to the white-noise limit. This is indeed a nontrivial task.

Since the Stratonovich drift can drastically modify the behavior of systems, and since it may not always be obvious what the appropriate noise prescription is in a given problem, it is particularly important to distinguish which noise effects are intrinsic, in the sense of occurring regardless the noise interpretation, and which ones are strictly associated to the Stratonovich drift. In other words, it is important to elucidate when the noise interpretation may only affect the quantitative behavior, and when it may indeed change the problem at a qualitative level.

For the case of noise-induced phase transitions, the noise prescription used so far in the literature is that of Stratonovich. Nevertheless, it could be argued that if the noise has an internal origin, one should, in principle, expect Itô noise, too, so it would be good to establish whether, in the latter case, 
noise-induced transitions can occur. We will see that this is indeed the case for a recently discovered class of noiseinduced phase transitions. From a theoretical point of view, it is also important to deal with Itô noise since then the continuum white-noise limit is either well defined or less singular than in the Stratonovich case [7]. This has important consequences in order to establish when the macroscopic observables will carry out a nontrivial, singular dependence on the spatial cutoff of the noise (Stratonovich case) and when such residual dependence will be weak (Itô case) [8].

Few contributions have appeared in the physics literature on Itô calculus in extended systems. A comparative discussion about the mathematical problems involved in the two interpretations appeared in Ref. [9]. The role of the multiplicative noise in the Itô interpretation has been analyzed in the context of spatiotemporal intermittency [10] and front propagation [11]. Dynamical renormalization group calculations were presented in Ref. [12]. However, noise-induced ordering phase transitions had been reported so far only in the framework of the Stratonovich interpretation [1-3]. In that case, the mechanism underneath these transitions is that the multiplicative noise term has a nonzero average value, which produces a short-time instability of the disordered phase and induces the ordered phase to arise $[2,3,13]$. The instability can be linear $[2,14]$ or nonlinear $[3,15]$, but is in any case induced by the so-called Stratonovich shift. Due to the absence of such a drift, the Itô interpretation does not present this type of noise-induced ordered phase, or any other spatially ordered state [16].

Recently, however, a new type of noise-induced phase transition has been found which does not occur via an instability of the disordered phase [17]. Here, the ordered phase arises due to the balance between the relaxing deterministic forces pushing the system toward the disordered state, and the activating multiplicative fluctuations pulling the field away from that state, in a type of entropy-driven phase transition (EDPT). This behavior is the spatiotemporal extension of noise-induced transitions in purely temporal, zerodimensional systems, where the probability distribution of the time-dependent variable exhibits a change in the number and type of its extrema as noise intensity varies [18]. A key idea in the model studied here is that the bimodality in the stationary probability density is not associated to a potential barrier, but has a dynamical origin. In fact, the dependence of the multiplicative noise term on the field is such that, for sufficiently large noise strength, the system escapes more easily from the central region than from the sides, despite the fact that the deterministic force always drives the system towards the center. As a result the peaks of the probability density are off-center. An important difference with the usual bimodality associated to a potential barrier is that in our case the characteristic relaxation time scales for the zerodimensional model are of order $1\left[\mathcal{O}\left(\varepsilon^{0}\right)\right]$ as opposed to $\mathcal{O}(\exp (1 / \varepsilon))$ which is characteristic of activation processes, $\varepsilon$ being a generic measure of the noise strength. In the spatially extended case, the spatial (diffusive) coupling of the field introduces an additional crucial ingredient, namely, it freezes the domains impeding the fast relaxation process of the zero-dimensional case. This gives rise to a well-defined, stable interface which then drives the much slower domaingrowth dynamics. Since no Stratonovich drift is required to induce this effect (as opposed, for instance, to the case of Ref. [1] where it takes the form of an effective barrier) it is to be expected that the corresponding class of model exhibiting this behavior should also display noise-induced ordering in the Itô interpretation. In this paper we show that this is indeed the case, by comparing the behavior of the model introduced in Ref. [17] for both the Itô and Stratonovich interpretations with that of a standard Ginzburg-Landau model with multiplicative noise (Sec. II). We also analyze in detail the dynamical properties of the growth processes arising from the noise-induced ordering transitions in the two cases (Sec. III), which will be shown to share universal characteristics (i.e., growth exponents) but differ in nonuniversal features (such as power-law prefactors). Finally, algorithms that have been specially developed for generating the results presented in this paper, for both the Stratonovich and Itô interpretations, are described in detail in the Appendix.

\section{THEORETICAL ANALYSIS}

We will use a model of a class of systems for which the steady-state probability distribution can be obtained exactly. As a consequence, the existence of a phase transition in these kinds of systems can be studied without any dynamical reference.

Our model corresponds to a relaxational flow in a freeenergy potential $\mathcal{F}(\{\phi\})$, with a field-dependent kinetic coefficient $\Gamma(\phi)$ and a fluctuating term fulfilling a fluctuationdissipation relation [17]. The model is defined by the following stochastic partial differential equation:

$$
\frac{\partial \phi(\vec{x}, t)}{\partial t}=-\Gamma(\phi(\vec{x}, t)) \frac{\delta \mathcal{F}}{\delta \phi(\vec{x}, t)}+\Gamma(\phi(\vec{x}, t))^{1 / 2} \xi(\vec{x}, t) .
$$

We suppose that the noise $\xi(\vec{x}, t)$ is Gaussian, with zero mean and correlation

$$
\left\langle\xi(\vec{x}, t) \xi\left(\vec{x}^{\prime}, t^{\prime}\right)\right\rangle=2 \sigma^{2} \delta\left(\vec{x}-\vec{x}^{\prime}\right) \delta\left(t-t^{\prime}\right),
$$

where $\sigma^{2}$ is the noise intensity. Moreover, we choose the following form for the free-energy potential $\mathcal{F}$,

$$
\mathcal{F}=\int d^{d} \vec{x}\left\{V_{0}(\phi(\vec{x}, t))+\frac{D}{4 d}[\vec{\nabla} \phi(\vec{x}, t)]^{2}\right\} .
$$

Since we are dealing with spatially uncorrelated noise, we perform the analysis in a discrete space in order to avoid singularities [9]. In a $d$-dimensional square lattice of mesh size $\Delta x$ and $N=L^{d}$ cells, our model reads

$$
\frac{d \phi_{i}}{d t}=-\Gamma_{i} \frac{\partial F}{\partial \phi_{i}}+\Gamma_{i}^{1 / 2} \xi_{i}(t),
$$

where only one index is used to label the cells, $\phi_{i} \equiv \phi\left(\vec{x}_{i}\right)$, $\Gamma_{i} \equiv \Gamma\left(\phi_{i}\right)$, and the noise satisfies the correlation 


$$
\left\langle\xi_{i}(t) \xi_{j}\left(t^{\prime}\right)\right\rangle=2 \sigma^{2} \frac{\delta_{i j}}{\Delta x^{d}} \delta\left(t-t^{\prime}\right)
$$

In discrete space, the free energy has the form

$$
F(\{\phi\})=\sum_{i=1}^{N}\left[V_{0}\left(\phi_{i}\right)+\frac{D}{4 d \Delta x^{2}} \sum_{j \in n n^{+}(i)}\left(\phi_{j}-\phi_{i}\right)^{2}\right],
$$

where the gradient term is approximated by the sum over nearest neighbors on the lattice in a standard way, $|\vec{\nabla} \phi|^{2}$ $\rightarrow \sum_{j \in n n^{+}{ }_{(i)}}\left(\phi_{j}-\phi_{i}\right)^{2} / \Delta x^{2}$, and $n n^{+}(i)$ stands for the $d$-nearest neighbors of $i$ in the positive direction of each axis. For simplicity, we choose a monostable local potential

$$
V_{0}(\phi)=\frac{a}{2} \phi^{2}
$$

where $a>0$. Finally, the kinetic coefficient $\Gamma(\phi)$ is taken to depend on the field in the following way [17]:

$$
\Gamma(\phi)=\frac{1}{1+c \phi^{2}}
$$

This functional dependence of the kinetic coefficient favors diffusion due to fluctuations in the disordered state.

Our objective now is to study Eq. (4) in the Stratonovich and Itô stochastic interpretations. The corresponding FokkerPlanck equation for the probability density of the field $P(\{\phi\}, t)$ can be written in a unified notation for both interpretations [5],

$$
\frac{\partial P}{\partial t}=\sum_{i} \frac{\partial}{\partial \phi_{i}}\left[\Gamma_{i} \frac{\partial F}{\partial \phi_{i}} P+\frac{B \sigma^{2}}{\Delta x^{d}} \Gamma_{i}^{1 / 2} \frac{\partial \Gamma_{i}}{\partial \phi_{i}} P+\frac{\sigma^{2}}{\Delta x^{d}} \frac{\partial}{\partial \phi_{i}} \Gamma_{i} P\right],
$$

where $B=1$ for the Stratonovich interpretation and $B=2$ in the Itô case.

If no probability flux is present, the stationary solution $P_{\text {st }}$ of Eq. (9) satisfies

$$
\left(\frac{\partial F}{\partial \phi_{i}}+\frac{B \sigma^{2}}{2 \Delta x^{d}} \frac{\partial \ln \Gamma_{i}}{\partial \phi_{i}}\right) P_{\mathrm{st}}+\frac{\sigma^{2}}{\Delta x^{d}} \frac{\partial P_{\mathrm{st}}}{\partial \phi_{i}}=0 .
$$

The solution of this equation is

$$
P_{\mathrm{st}}(\{\phi\}) \sim \mathrm{e}^{-F_{\mathrm{eff}} \Delta x^{d} / \sigma^{2}},
$$

where we have introduced the effective free energy

$$
F_{\text {eff }}(\{\phi\}) \equiv F(\{\phi\})+\frac{B \sigma^{2}}{2 \Delta x^{d}} \sum_{i=1}^{N} \ln \Gamma_{i} .
$$

The above expressions can be written in continuum space as

$$
P_{\text {st }}(\{\phi\}) \sim \mathrm{e}^{-\mathcal{F}_{\text {eff }} / \sigma^{2}},
$$

$$
\mathcal{F}_{\text {eff }}^{S}(\{\phi\}) \equiv \mathcal{F}(\{\phi\})+\frac{B \sigma_{0}^{2}}{2} \int d^{d} x \ln \Gamma(\phi(\vec{x})),
$$

where $\sigma_{0}^{2} \equiv \sigma^{2} / \Delta x^{d}$ stands for the effective noise intensity of a spatially white noise in a discrete space.

We have thus seen that the stationary multivariate probability distribution can be obtained exactly in both the Ito and Stratonovich interpretations for the spatially extended EDPT model, and that both lead to very similar qualitative results. The only difference is an extra factor 2 in the new term of the effective potential in the Ito interpretation. As is already known [17], the EDPT model presents a continuous ordering noise-induced phase transition in the Stratonovich interpretation. But according to the results shown above, and as will be shown in the following section, this model also exhibits an ordering transition in the Ito interpretation, although the location of the critical point will be different. We should remark here that, as in the case of the Stratonovich interpretation [17], this phase transition is not due to a shorttime instability of the homogeneous null phase. Indeed, the linear equation for the first statistical moment $\langle\phi\rangle$ can be computed to be [1]

$$
\frac{\partial\langle\phi\rangle}{\partial t}=-\left[a+(2-B) \sigma_{0}^{2} c\right]\langle\phi\rangle+\frac{D}{2 d} \nabla^{2}\langle\phi\rangle .
$$

For $a>0$, the homogeneous null solution of this equation is stable for all noise intensities, both for $B=1$ and $B=2$. Therefore, the mechanism of this phase transition must be different from the standard one.

\section{STEADY-STATE BEHAVIOR}

A standard way of determining the existence of a noiseinduced phase transition is by applying a mean-field approximation to the Langevin or Fokker-Planck equations of the system [1,2]. In the present case, however, since we have obtained the exact multivariate probability distribution in both interpretations, we will implement that approximation directly on the effective potential derived from Eq. (12).

The mean-field approximation consists of replacing the exact value of the neighbor field in the Langevin or FokkerPlanck equation by a common mean-field value $\langle\phi\rangle$. In the present case, we make such an identification in the neighboring values of the gradient term appearing in the effective free energy [see Eqs. (6) and (12)]:

$$
\frac{1}{\Delta x^{2}} \sum_{j \in n n^{+}(i)}\left(\phi_{j}-\phi_{i}\right)^{2} \approx \frac{2 d}{\Delta x^{2}}\left(\langle\phi\rangle-\phi_{i}\right)^{2} \text {. }
$$

In this way, the effective free energy becomes

$$
\begin{aligned}
F_{\mathrm{eff}}(\{\phi\},\langle\phi\rangle)= & \sum_{i=1}^{N}\left\{V_{0}\left(\phi_{i}\right)+\frac{B \sigma_{0}^{2}}{2} \ln \Gamma\left(\phi_{i}\right) \frac{D}{2 \Delta x^{2}}\right. \\
& \left.\times\left(\phi_{i}-\langle\phi\rangle\right)^{2}\right\} \equiv \sum_{i=1}^{N} V_{\mathrm{eff}}\left(\phi_{i},\langle\phi\rangle\right) .
\end{aligned}
$$




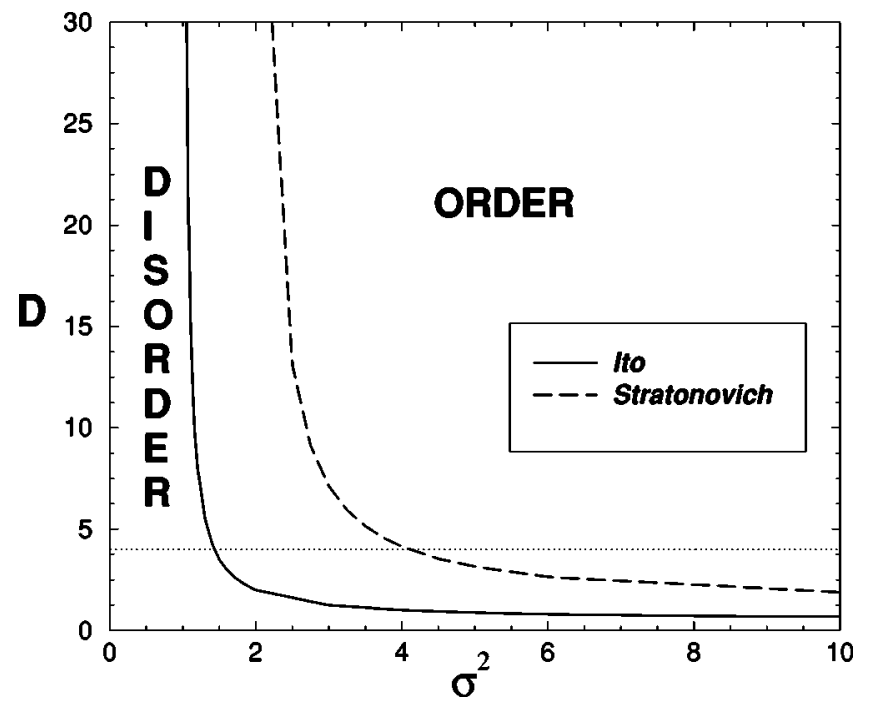

FIG. 1. Phase diagram of the EDPT model, obtained from a mean-field analysis, in the Itô (continuous line) and Stratonovich (dashed line) interpretations. The horizontal dotted line corresponds to the value of $D$ used in Fig. 2. The parameter values are $a=1$, $c=0.5$, and $\Delta x=1$.

The unknown mean-field value $\langle\phi\rangle$ is obtained selfconsistently, according to

$$
\langle\phi\rangle=\int_{-\infty}^{\infty} \phi P_{\mathrm{st}}(\phi,\langle\phi\rangle),
$$

where the one-site probability distribution $\left(P_{\mathrm{st}}(\{\phi\})\right.$ $\left.=\prod_{i=1}^{N} P_{\mathrm{st}}\left(\phi_{i}\right)\right)$ is given by

$$
P_{\mathrm{st}}(\phi) \sim \mathrm{e}^{-V_{\mathrm{eff}} / \sigma_{0}^{2}}
$$

The mean-field predictions for $\langle\phi\rangle$ in the two-dimensional case are plotted in Fig. 1, where lines separating the situations where $\langle\phi\rangle=0$ (disorder) and $\langle\phi\rangle \neq 0$ (order) are plotted for both the Itô and Stratonovich interpretations in the space of parameters $D$ and $\sigma^{2}$. The figure shows that both interpretations predict a continuous noise-induced ordering phase transition, which occurs earlier (i.e., for lower noise intensities) in the Itô case. In particular, in the large coupling limit $(D \rightarrow \infty)$ the transition in the Itô interpretation takes place at a critical noise intensity $\left(\sigma_{c}^{2}=a / B c\right.$, for $\left.\Delta x=1\right)$, that is half the critical value in the Stratonovich case, both of which coincide with the transition point in zero-dimensional systems (with noise intensity $\sigma^{2} / \Delta x^{2}$ ) [18].

Note that, in contrast with the usual noise-induced transitions (which exhibit reentrant phenomena), the transition lines of Fig. 1 decay monotonically with $\sigma^{2}$. This implies, therefore, that no minimum coupling strength is required in these models for a phase transition to occur.

In order to validate the results obtained from the meanfield approximation, we have performed extensive numerical simulations of model (4)-(8) in both the Itô and Stratonovich interpretations. To that end, we have developed a new type of numerical algorithm suitable for the implementation of both stochastic interpretations of the multiplicative noise. The

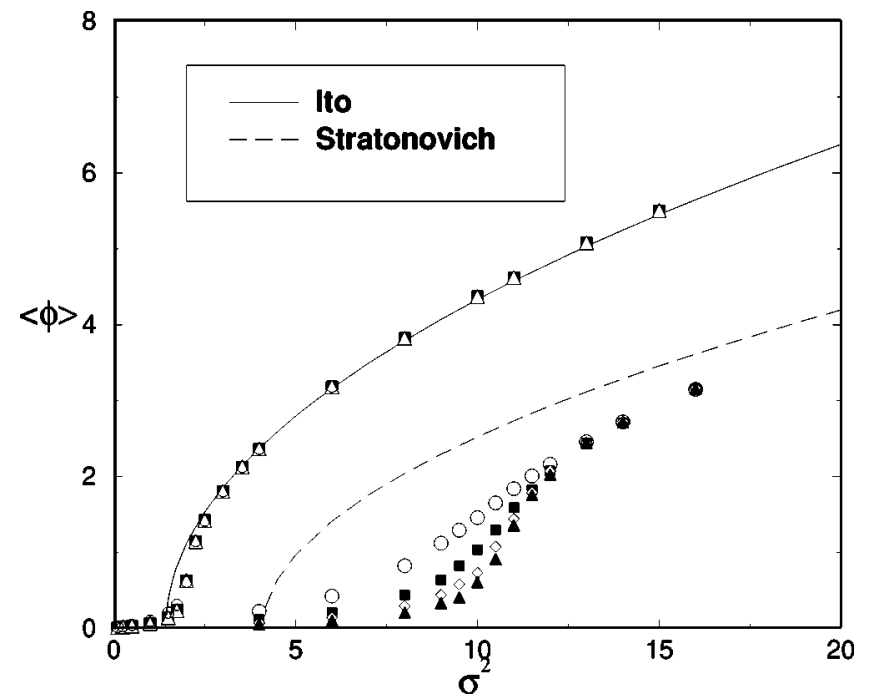

FIG. 2. Mean-field and numerical simulation results for the EDPT model in the Itô (continuous line) and Stratonovich (dashed line) interpretations. Simulations have been performed for different system sizes: $L=16$ (circles), $L=24$ (squares), and $L=32$ (triangles) for Itô, and $L=64$ (triangles), $L=48$ (diamonds), $L=32$ (squares), and $L=16$ (circles) for Stratonovich. $D=4$, and the rest of parameter values are those of the previous figure.

derivation of this algorithm and a comparison with the wellknown Heun algorithm (derived only for the Stratonovich interpretation) is presented in the Appendix. The simulations have been performed on a square lattice of $256 \times 256$ cells of mesh size $\Delta x=1$, with a time step $\Delta t=0.01$ and periodic boundary conditions (except when explicitly indicated). Where necessary, we have averaged over 10 realizations of the noise and the initial random conditions, corresponding to Gaussian or uniform distributions. In order to compute the mean field, we first evaluate the spatial average of the system:

$$
\langle\phi(t)\rangle=\frac{1}{N}\left|\sum_{i=1}^{N} \phi_{i}(t)\right|,
$$

where $N$ is the number of lattice cells, and $\phi_{i}(t)$ is the field value at the $i$ cell. Once the spatial average reaches a stationary state, the temporal average is evaluated as

$$
\langle\phi\rangle=\frac{1}{T_{M}-T_{m}} \sum_{t=T_{m}}^{T_{M}}\langle\phi(t)\rangle,
$$

where $T_{M}$ and $T_{m}$ delimit the time interval within the steadystate regime in which the temporal average is calculated. Afterwards, the realization average can be computed.

The numerical simulation results for the two interpretations are shown in Fig. 2, where they are also compared with the predictions coming from the mean-field approximation. Due to the value of $D$ chosen, the agreement between the mean-field estimate and the simulations is better for the Ito interpretation. In any case, the model exhibits a noiseinduced ordering phase transition for both interpretations, as predicted by the mean-field approach. 


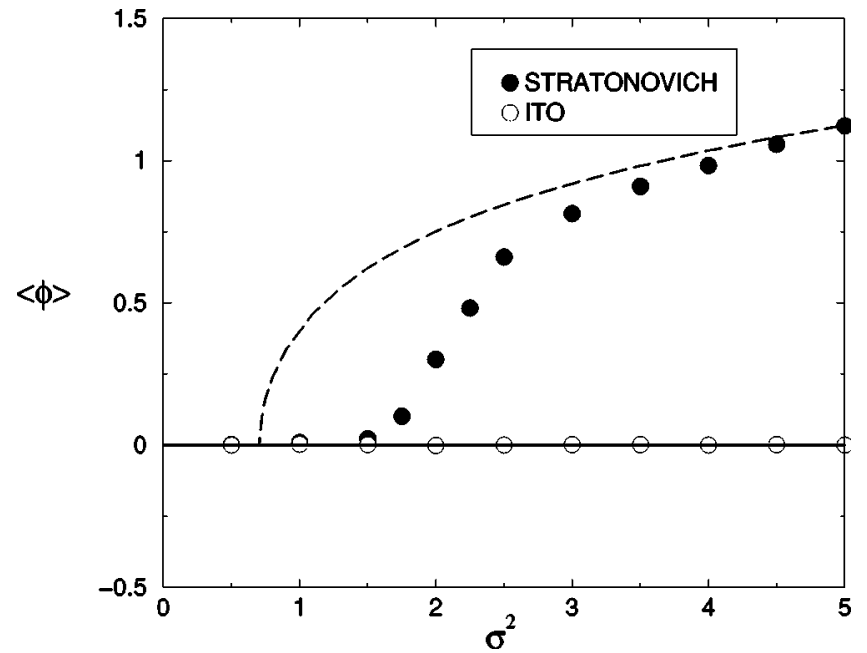

FIG. 3. Bifurcation diagram of the Ginzburg-Landau model in the Itô and Stratonovich interpretations. Both mean-field and 2D simulation results are shown. The parameters used are $L=30, a=$ $-0.2, b=1, D=4$, and the additive noise intensity 0.5 .

We stress at this point that standard models exhibiting noise-induced phase transitions caused by short-term instabilities of the disordered phase do so only in the case of the Stratonovich interpretation. In order to illustrate this point, we present here for comparison what happens in the wellknown case of the Ginzburg-Landau model with external multiplicative fluctuations [3]:

$$
\frac{d \phi}{d t}=a \phi-b \phi^{3}+D \nabla^{2} \phi+\phi \xi(\vec{x}, t)+\eta(\vec{x}, t),
$$

where $\eta(\vec{x}, t)$ and $\xi(\vec{x}, t)$ are Gaussian and white noises. This system presents a noise-induced phase transition if we interpret the noise in the Stratonovich sense, but not if one uses the Itô interpretation. This can be seen in Fig. 3, where the two simulations share the same conditions and parameters. In the Itô interpretation the ordered parameter $\langle\phi\rangle$ remains always in the disordered state, due to the fact that the noisedependent drift that causes the short-time instability is only present in the the Stratonovich prescription [16].

\section{DOMAIN GROWTH DYNAMICS}

We have seen that the EDPT model in the presence of external fluctuations can reach a stationary ordered state described by a nonzero order parameter $\langle\phi\rangle$, for both the Itô and Stratonovich interpretations. This means that, if the system is initially in a disordered steady state $\langle\phi\rangle=0$ corresponding to a small noise intensity, as the intensity of external fluctuations is increased above its critical value the system develops domains of the two new symmetric stationary ordered phases, that grow with time as shown in Fig. 4. The figure shows that the system behaves differently in the two stochastic interpretations for the same noise intensity, the Itô case being much more contrasted due to the fact that the order parameter is larger than in the Stratonovich case, which is very noisy.
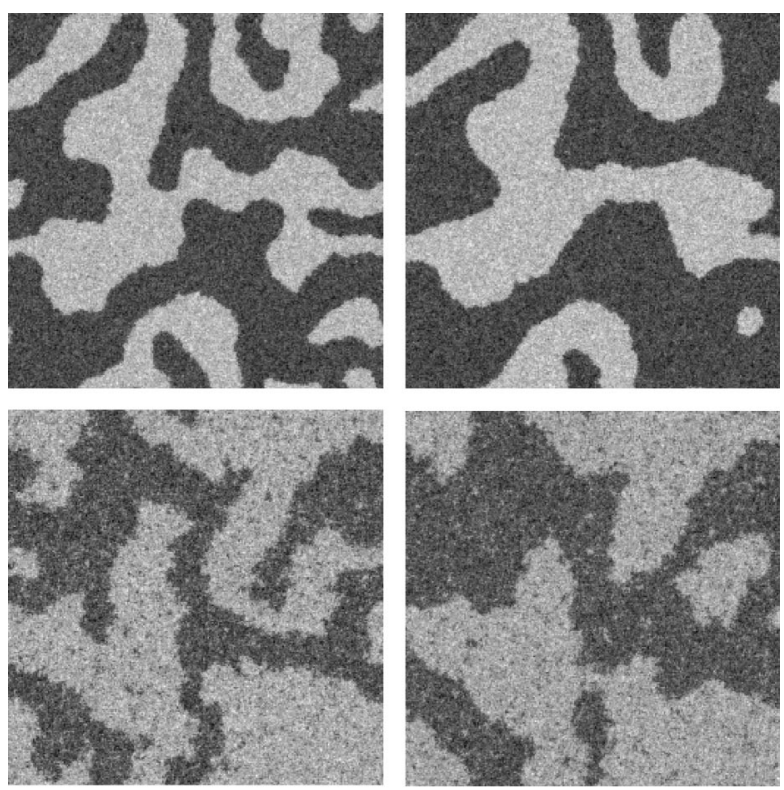

FIG. 4. Snapshots of evolving noise-induced domains for the EDPT model at $t=750$ (left figures) and $t=1750$ (right figures) in the Itô (top) and Stratonovich (bottom) interpretations. Parameters are $a=1, c=3, \sigma^{2}=3.5$, and $L=256$.

In this section we are concerned with the growth of these noise-induced domains. Although the mechanism that induces the phase transition is different from those that have been reported before, we can expect that, once the domains have appeared, their dynamics has the same characteristics as those of the domain growth following the quench of a system below its order-disorder transition temperature, as happens in the Ginzburg-Landau model [19].

For nonconserved order parameter models, one of the domains grows until it fills the whole system. The mechanism underlying domain growth in this case is the motion of the interface between domains caused by the interface structure. The translational velocity of the domain boundary has been found to be proportional to the mean curvature of the boundary, and independent of the free energy of the interface. This can be quantified by the equation of motion obeyed by the characteristic length (i.e., the average radius) of the domains of equilibrium phases, $R(t)$ [20],

$$
\frac{d R}{d t}=A \frac{\Gamma}{R},
$$

where $A$ is a model-dependent constant and $\Gamma$ is the kinetic coefficient multiplying the diffusion term. This expression leads in a straightforward way to the Allen-Cahn law of domain growth,

$$
R(t) \propto \sqrt{2 A \Gamma} t^{1 / 2}
$$

In the time regime where this law is verified, $R(t)$ is the only characteristic length of the system, and a scaling behavior for its spatial structure at different times is found. All these results are known to apply also in the case of standard noiseinduced phase transitions caused by linear instabilities of the 


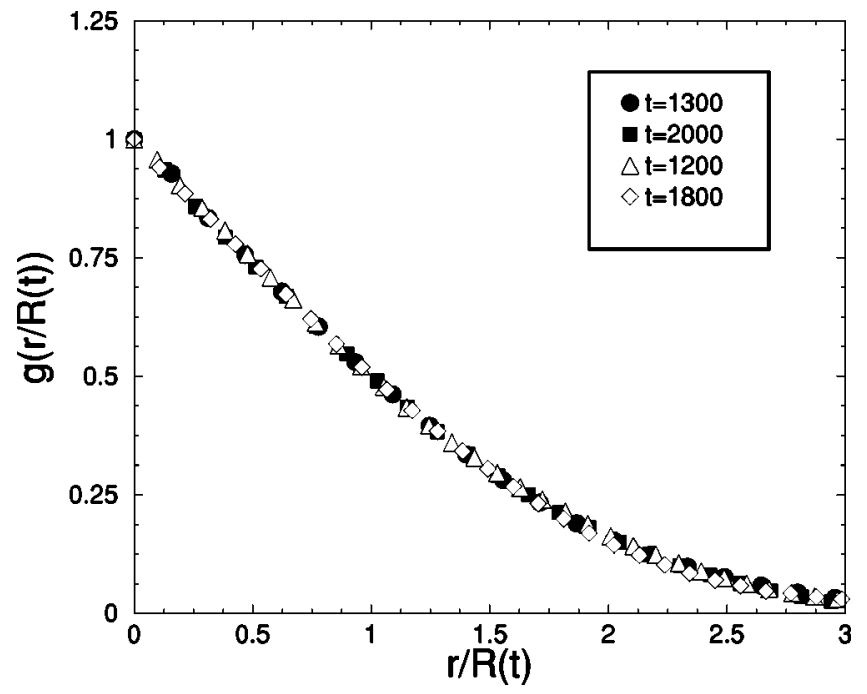

FIG. 5. Scaled pair correlation function for the EDPT model in the Stratonovich interpretation for $t=1300$ (circles) and $t=2000$ (squares), and in the Itô interpretation for $t=1200$ (triangles) and $t=1800$ (diamonds). The parameter values are $a=1, c=3, D$ $=4, \sigma^{2}=3.5$, and $\Delta x=1$.

homogeneous disordered phase [14]. We want to find out whether the same thing happens in the EDPT described in this paper.

In order to characterize the dynamics of model (4), we let our system evolve from an initial disordered state, and compute the isotropic correlation $G(r, t)$ function at different times. We use the following normalization:

$$
g(r, t)=\frac{G(r, t)}{G(0, t)} .
$$

Let us consider a time regime in which there is only one characteristic length $R(t)$ in the system, which is related to the average size of the domains. There are several possible definitions for $R(t)$, but all of them should lead to the same results. We have chosen $R(t)$ as the distance at which $g(r, t)$ has half its maximum value. In this time regime, we can apply the scaling hypothesis for a $d$-dimensional system,

$$
g(r, t)=g(r / R(t)),
$$

with no other explicit time dependence. When these relations hold, the spatial structure of the system at different times is statistically equivalent, except for a scale factor. Since the domain growth is more clearly observed far from the critical point, we have taken new parameter values accordingly. The numerical results in the Stratonovich interpretation for the scaled pair correlation function are represented in Fig. 5. As shown in Ref. [19], the pair correlation function exhibits a discontinuity in its first derivative in the presence of noise sources. We have eliminated this discontinuity by fitting a parabolic function in the origin $(r=0, \Delta x)$. The same study has been made in the case of Ito interpretation under the same conditions and parameters.

We now compare the temporal evolution of the characteristic length of the system $R(t)$ for the two stochastic inter-

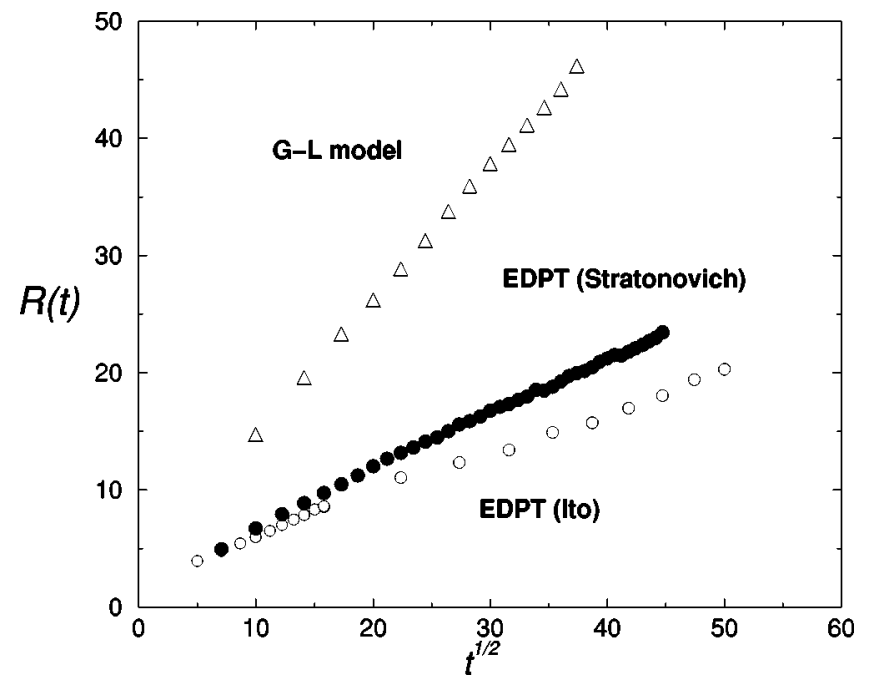

FIG. 6. Allen-Cahn law for the GL model (same parameter values as in Fig. 3 with $\epsilon=10^{-4}$ and $\sigma^{2}=0.6$ ) and the EDPT model for equal noise intensities (and different mean fields). The latter is computed in both the Itô and Stratonovich interpretations. The parameter values for the EDPT model are $a=1, c=3, D=4, \sigma^{2}$ $=3.5$, and $\Delta x=1$.

pretations. Figure 6 presents this comparison for equal values of the noise intensity. From these numerical results we can conclude that the Allen-Cahn law is satisfied for the two interpretations, and that there is a time regime in which the system is self-similar. One interesting fact is that domain evolution in Itô is slower than in Stratonovich, and in both cases much slower than the Ginzburg-Landau model. This fact can be explained looking at the constant prefactor $\sqrt{2 A \Gamma}$ of the Allen-Cahn law (24). In the Ginzburg-Landau model $\Gamma=1$, but in the EDPT model this quantity is field dependent (8), and can be approximated by

$$
\Gamma \approx \frac{1}{1+c\left\langle\phi^{2}\right\rangle} \approx \frac{1}{1+c\langle\phi\rangle^{2}} .
$$

According to this expression, and since for a fixed $\sigma^{2}$ we have that $\left.\langle\phi\rangle_{I}\right\rangle\langle\phi\rangle_{S}$, as a consequence we should expect the slowest growth for the Ito EDPT case, and the fastest one for the Ginzburg-Landau model. This is what we can see in Fig. 6.

In order to eliminate the influence of the stationary meanfield value $\langle\phi\rangle$ on the growth rate, we have compared the evolution of the system under the two interpretations using in each case a different noise intensity, so that the mean field has the same value in the two cases. The results are shown in Fig. 7, where we have fixed $\langle\phi\rangle=3.15$ for the two interpretations, for which we need $\sigma^{2}=16$ in the Stratonovich interpretation and $\sigma^{2}=6$ in the Itô interpretation. As can be seen, in both interpretations the system seems to evolve at the same rhythm, although the slope in Itô is slightly higher than in Stratonovich. We think that this small difference is due to the fact that although $\langle\phi\rangle$ is the same for both interpreta- 


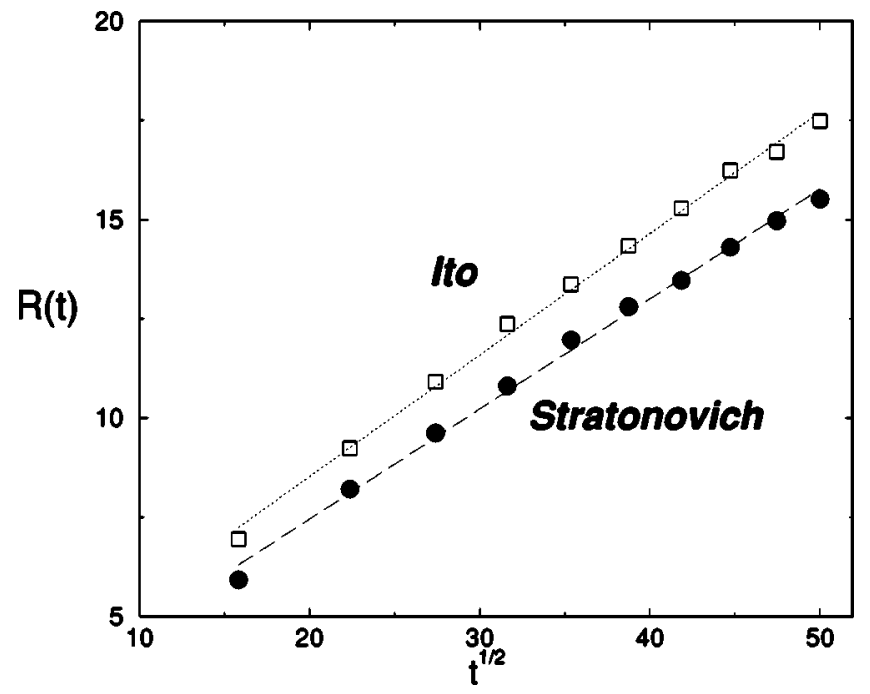

FIG. 7. Allen-Cahn law for the EDPT model for equal mean fields (and different noise intensities), under both the Itô and Stratonovich interpretations. For Stratonovich $\sigma^{2}=16$, for Itô $\sigma^{2}=6$. Other parameter values are $a=1, c=0.5$.

tions, the Stratonovich case is much more fluctuating, and hence we have to expect a larger $\left\langle\phi^{2}\right\rangle$ and accordingly a lower slope.

\section{COMMENTS AND CONCLUSIONS}

It is worth commenting here that an effective model can be developed which has the same stationary solutions as the EDPT model, but different dynamics. The dynamical equation for this effective model with $\Delta x=1$ is

$$
\frac{\partial \phi}{\partial t}=-a \phi+\frac{B \sigma^{2} c \phi}{1+c \phi^{2}}+\frac{D}{2 d} \nabla^{2} \phi+\xi(\vec{x}, t)
$$

where, as before, $B$ is a parameter whose value indicates the interpretation that we are mimicking $(B=1$ for Stratonovich and $B=2$ for Itô). The correlation of noise is given by Eq. (2). The equation of motion of the mean value of the field in the linear approximation is

$$
\frac{d\langle\phi\rangle}{d t}=\left(B \sigma^{2} c-a\right)\langle\phi\rangle+\frac{D}{2 d} \nabla^{2}\langle\phi\rangle,
$$

which tells us that, for $\sigma^{2}>a / B c$, the homogeneous phase $\langle\phi\rangle=0$ is unstable. This instability does not appear in the EDPT model [see Eq. (15)]. According to this result, we have to expect an initial transient faster in this model (as in the Ginzburg-Landau model) than in the EDPT cases. This fact has been checked numerically and it can be seen in Fig. 8. We can clearly appreciate that the effective model has a much faster initial transient than the EDPT model, for the same values of the parameters which is a signature of the different character of the instability of the initial state. While this observation applies also to the zero-dimensional version of the model, the crucial ingredient in our EDPT model is the role of the spatial coupling, which prevents the fast transition

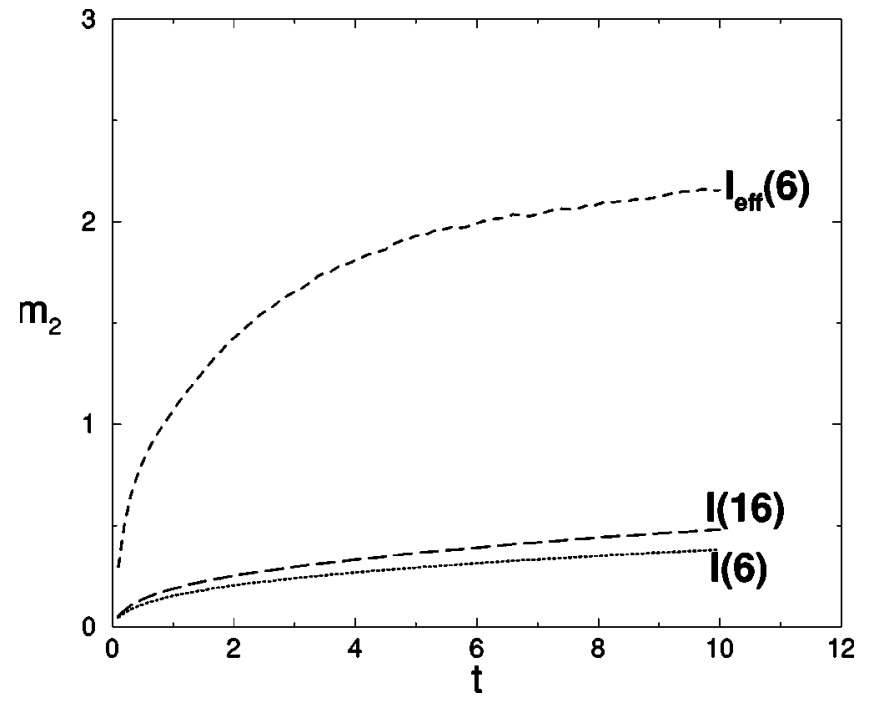

FIG. 8. Transient evolution of the quantity $m_{2}=\left\langle\phi^{2}(t)\right\rangle /\langle\phi\rangle_{s t}^{2}$ which measures the emergence of order from homogeneous initial condition $\phi=0$. The letter $I$ means the Itô case and the value of the intensity of the noise is inside the parentheses.

between the two probability peaks. The fact that this transition occurs in a deterministic time scale in the zerodimensional case is what distinguishes the problem from the usual, barrier-crossing bistability. In the spatially extended case, however, it is precisely the spatial coupling what generates an effective barrier allowing for the formation of stable domains, with an interface-driven dynamics.

In conclusion, we have presented a nonequilibrium field model for which one can compute exactly the stationary probability distribution, and which exhibits an intrinsic noise-induced ordering phase transition irrespective of the stochastic interpretation of the multiplicative noise term. In particular, the phase transition is found in the Itô interpretation, where so far, noise had only been seen to have a disordering effect. The same model can be studied changing the diffusive term by the spatial coupling of the SwiftHohenberg model in which case a noise-induced pattern transition is found [21]. These types of models do constitute a generalization of the Horsthemke-Lefever noise-induced transitions to genuine noise induced phase transitions in extended systems.

\section{ACKNOWLEDGMENTS}

This research was supported by the Dirección General de Enseñanza Superior (Spain) under Project Nos. BFM20000624, BFM2001-2159, BXX2000-0638-C02-02 and from the Generalitat de Catalunya (Project No. 2001SGR00223).

\section{APPENDIX: STOCHASTIC ALGORITHMS}

Here we will derive an alternative algorithm that is an extension of the well-known Heun algorithm, valid for both the Itô and Stratonovich interpretations of stochastic differential equations with multiplicative noise. Our aim is to simulate numerically the following stochastic differential 
equation on a $d$-dimensional lattice,

$$
\frac{\partial \phi(\vec{x}, t)}{\partial t}=f(\phi(\vec{x}, t), \nabla)+g(\phi(\vec{x}, t)) \xi(\vec{x}, t) .
$$

First of all, we write this equation in a discrete space as follows:

$$
\frac{d \phi_{i}(t)}{d t}=f_{i}(\phi(t))+g_{i}(\phi(t)) \xi_{i}(t)
$$

where $i$ stands for the position inside the lattice, and the noise correlation is given by Eq. (5).

The first step in the derivation of the algorithm is to integrate formally Eq. (A2) to get

$$
\begin{aligned}
\phi_{i}(t+\Delta t)= & \phi_{i}(t)+\int_{t}^{t+\Delta t} f_{i}\left(\phi\left(t^{\prime}\right)\right) d t^{\prime} \\
& +\int_{t}^{t+\Delta t} g\left(\phi\left(t^{\prime}\right)\right) \xi\left(t^{\prime}\right) d t^{\prime} .
\end{aligned}
$$

The first integral in Eq. (A3) is evaluated according to a second-order predictor-corrector algorithm,

$$
\begin{aligned}
\phi_{i}(t+\Delta t)= & \phi_{i}(t)+\frac{f_{i}(\phi(t))+f_{i}(\widetilde{\phi}(t))}{2} \Delta t \\
& +\int_{t}^{t+\Delta t} g\left(\phi\left(t^{\prime}\right)\right) \xi\left(t^{\prime}\right) d t^{\prime},
\end{aligned}
$$

where $\widetilde{\phi}(t)$ is the predictor term defined as the first-order solution of Eq. (A3),

$$
\widetilde{\phi}_{i}(t)=\phi_{i}(t)+f_{i}\left(\phi_{i}(t)\right) \Delta t+g_{i}(\phi(t)) X_{i}
$$

This expression defines the first equation of the algorithm, which is independent of the stochastic interpretation. $X_{i}(t)$ is the Wiener process, defined as

$$
X_{i}(t)=\int_{t}^{t+\Delta t} \xi_{i}\left(t^{\prime}\right) d t^{\prime}
$$

and whose numerical implementation is

$$
X_{i}(t)=\sqrt{\frac{2 \sigma^{2} \Delta t}{\Delta x^{2}}} \alpha_{i}
$$

where $\alpha_{i}$ are independent Gaussian random numbers of zero mean and unity variance, and they are implemented using Ref. [22].

The second integral in Eq. (A3) is not well defined, and one needs to make a prescription for its evaluation, at least up to first order in $\Delta t$.

The standard Heun algorithm works for the Stratonovich interpretation, and makes the following assumption:

$$
\int_{t}^{t+\Delta t} g\left(\phi\left(t^{\prime}\right)\right) \xi\left(t^{\prime}\right) d t^{\prime}=\left(\frac{g\left(\phi_{i}(t)\right)+g\left(\widetilde{\phi}_{i}(t)\right)}{2}\right) X_{i}(t) .
$$

Accordingly, the second equation of this algorithm is

$$
\begin{aligned}
\phi_{i}(t+\Delta t)= & \phi_{i}(t)+\frac{f_{i}(\phi(t))+f_{i}(\widetilde{\phi}(t))}{2} \Delta t \\
& +\left(\frac{g\left(\phi_{i}(t)\right)+g\left(\widetilde{\phi}_{i}(t)\right)}{2}\right) X_{i}(t) .
\end{aligned}
$$

On the other hand, in the Stratonovich calculus this integral is interpreted as [5]

$$
\int_{t}^{t+\Delta t} g\left(\phi\left(t^{\prime}\right)\right) \xi\left(t^{\prime}\right) d t^{\prime}=g\left(\frac{\phi_{i}(t)+\widetilde{\phi}_{i}(t)}{2}\right) X_{i}(t),
$$

so that the second equation of the algorithm is

$$
\begin{aligned}
\phi_{i}(t+\Delta t)= & \phi_{i}(t)+\frac{f_{i}(\phi(t))+f_{i}(\widetilde{\phi}(t))}{2} \Delta t \\
& +g\left(\frac{\phi_{i}(t)+\widetilde{\phi}_{i}(t)}{2}\right) X_{i}(t),
\end{aligned}
$$

which is not exactly the standard Heun algorithm. This is the algorithm that has been used in this paper for the Stratonovich interpretation results. Its advantage is that, in contrast to the Heun algorithm, our method has an analog in the Ito interpretation, for which the same integral is defined as [5]

$$
\int_{t}^{t+\Delta t} g_{i}\left(\phi\left(t^{\prime}\right)\right) \xi_{i}\left(t^{\prime}\right) d t^{\prime}=g_{i}(\phi(t)) X_{i}(t)
$$

Therefore, the second equation of the algorithm in the Ito interpretation reads

$$
\phi_{i}(t+\Delta t)=\phi_{i}(t)+\frac{f_{i}(\phi(t))+f_{i}(\widetilde{\phi}(t))}{2} \Delta t+g_{i}(\phi(t)) X_{i}(t) .
$$

Given these results, the algorithm proceeds by evaluating first the predictor contribution (A5) and, using this value, computing the corrector term (A9), (A11) or (A13), corresponding to the Heun, Stratonovich, or Itô algorithms, respectively. All these three different algorithms are approximations up to the same order (second order in the deterministic part but first order in the stochastic one), when properly expanded in powers of $\Delta t$. One can check that there are no differences, up to these orders, between the Heun and Stratonovich algorithms, as it should be. Nevertheless, the Stratonovich prescription has an extra term, $1 / 2 g\left(\phi_{i}\right) g^{\prime}\left(\phi_{i}\right) X_{i}(t)^{2}$, with respect the Itô one, which is of order $\Delta t$. Our Itô algorithm also agrees with that presented in Ref. [23] up to order $\Delta t^{2}$. 
[1] J. García-Ojalvo and J.M. Sancho, Noise in Spatially Extended Systems (Springer, New York, 1999).

[2] C. Van den Broeck, J.M.R. Parrondo, and R. Toral, Phys. Rev. Lett. 73, 3395 (1994); C. Van den Broeck, J.M.R. Parrondo, R. Toral, and R. Kawai, Phys. Rev. E 55, 4084 (1997).

[3] J.M. Sancho and J. García-Ojalvo, in Lecture Notes in Physics, edited by J. Freund and T. Pöschel, (Springer, Berlin, 2000), Vol. 557.

[4] Additive noise has been seen to lead to order as well, provided multiplicative noise is also present; see, for instance, A. Zaikin and L. Schimansky-Geier, Phys. Rev. E 58, 4355 (1998).

[5] N.G. van Kampen, Stochastic Processes in Physics and Chemistry (North-Holland, Amsterdam, 1983).

[6] Ch. R. Doering, C. Mueller, and P. Smereka, Physica A (to be published).

[7] E. Alós, J.A. León, and D. Nualart, Probab. Theory Relat. Fields 115, 41 (1999).

[8] A. Rocco, L. Ramírez-Piscina, and J. Casademunt, Phys. Rev. E 65, 056116 (2002).

[9] C. Doering, Phys. Lett. A 122, 133 (1987).

[10] M.G. Zimmermann, R. Toral, O. Piro, and M. San Miguel, Phys. Rev. Lett. 85, 3612 (2000).

[11] J.M. Sancho and A. Sánchez, Eur. Phys. J. B 16, 127 (2000).

[12] Y. Tu, G. Grinstein, and M.A. Muñoz, Phys. Rev. Lett. 78, 274
(1997).

[13] A. Becker and L. Kramer, Phys. Rev. Lett. 73, 955 (1994).

[14] M. Ibañes, J. García-Ojalvo, R. Toral, and J.M. Sancho, in Lecture Notes in Physics, edited by J. Freund and T. Pöschel (Springer, Berlin, 2000), Vol. 557, p. 247.

[15] O. Carrillo, M. Ibañes, and J.M. Sancho, Fluct. Noise Lett. 2, L1 (2002).

[16] W. Genovese and M.A. Muñoz, Phys. Rev. E 60, 69 (1999).

[17] M. Ibañes, J. García-Ojalvo, R. Toral, and J.M. Sancho, Phys. Rev. Lett. 87, 020601 (2001).

[18] W. Horsthemke and R. Lefever, Noise-induced Transitions (Springer, Berlin, 1984).

[19] M. Ibañes, J. García-Ojalvo, R. Toral, and J.M. Sancho, Eur. Phys. J. B 18, 663 (2000).

[20] J.D. Gunton, M. San Miguel, and P.S. Sahni, in Phase Transitions and Critical Phenomena, edited by C. Domb and J.L. Lebowitz (Academic Press, New York, 1983), Vol. 8.

[21] J. Buceta, M. Ibañes, J.M. Sancho, and K. Lindenberg, Phys. Rev. E 67, 021113 (2003).

[22] R. Toral and A. Chakrabarti, Comput. Phys. Commun. 74, 4084 (1993).

[23] N.J. Rao, J.D. Borwankar, and D. Ramkrishna, SIAM J. Control 12, 124 (1974). 